\title{
Numerical investigation of side arm gas volume fraction in two phase $\mathbf{T}$-junction
}

\author{
Ahmed Saieed, Ban Sam, Wiliam Pao* and Fakhruldin M Hashim \\ Universiti Teknologi PETRONAS, 32610 Bandar Seri Iskandar, \\ Perak, Malaysia \\ *Email: william.pao@UTP.edu.my \\ Phone: +6053687012; Fax: +6053656461
}

\begin{abstract}
T-junction is commonly installed at the offshore production header as a separator to tap produced gas for downstream process operation. A major issue in the T-junction is the liquid carryover, where excessive liquid flows into the downstream process equipment causing the platform to trip. The primary objective of the present study is to determine the effect of ratio of side arm to main arm diameters on passive separation performance in T-junction. The analysis was carried out using Eulerian Multiphase model from ANSYS Fluent 16.1. The boundary conditions chosen for the inlet and two outlets of the T-junction were velocity inlet and volume fraction outlet flow, respectively. Likewise, pressure velocity coupling was achieved by using SIMPLE coupling scheme. Three different side to main arm diameter ratio and side to main arm length ratio of 0.2, 0.6, and 1.0 were investigated, with different gas velocities variation and inlet gas saturation. Investigation found that the gas volume fraction in the side arm cannot go beyond $90 \%$ irrespective of the variation of parameters such as gas to liquid velocity ratio, diameter ratio, length ratio, and mass split ratio. It was found that the amount of gas exiting through the side arm decreases by decreasing the initial gas saturation and gas to liquid velocity ratio. However, by decreasing the mass split ratio, more gas ends up in side arm. Likewise, in terms of geometrical aspects, increasing the $\mathrm{T}$-junction diameter ratio and reducing the length ratio causes an increase in extraction of gas into side arm. It was also noticed that the highest fraction of gas in side arm was achieved when the diameter ratio was 0.6. Therefore, it can be said that maximum amount of gas can be received in side arm when diameter ratio is around 0.6, initial gas saturation and velocity ratio are high, and mass split ratio is small. This paper discusses some of the most important parameters that are involved in partial phase separation in a T-junction. In order to have a more overall insight of the phase separation phenomena, it is required to explore the effects of inlet and outlet pressures, surface roughness of the pipe interior, angle between side and run arm, curvature radius at the junction, and the viscosity of the operating fluids.
\end{abstract}

Keywords: T-junction; liquid-gas separation; separation efficiency

\section{INTRODUCTION}

A stand-pipe appendage, or more commonly known as T-junction, is a very common geometric feature in pipe networks in the petroleum industry as it is the most common conduit used to divert fluid flow from one place to another before their final destination. T-junctions are famous for causing uneven distribution of the phases passing through them. Thus, industries have been taking advantage of this characteristic of the T-junction 
for decades, as it can be used as a partial phase separator. However, in order to get the most out of a simple T-junction, it is required to understand the parameters which affect the phase separation inside a $\mathrm{T}$-junction.

Understanding all the parameters which control the phase separation behaviour at the junction is a very complicated task as one has to understand eight different parameters regarding T-junction geometry alone in order to use it properly [1]. Similarly, flow parameters such as flow rate and operating pressure etc., and fluid properties such as density, viscosity, and surface tension also require serious consideration. It is due to the involvement of so many different variables that to this date, the phase separation phenomenon in a T-junction is quite unpredictable. As Lahey [2] said, there is not even a single numerical prediction model which can provide a reasonable prediction for phase separation for any new geometry and operating conditions, despite numerous experimental and numerical studies.

One of the main causes of the phase separation at the junction is because of the density difference of the operating phases [3]. It is much easier for the phase with lower density (gas) to change its direction and turn into side arm as compared to the phase with higher density (liquid). Hence, each of them takes their own route ultimately causing partial phase separation at the junction. Another parameter which plays a vital role in phase separation is gravitational force. By changing the orientation of the side arm with respect to the horizontal plane of reference, the way in which gravity affects the phases at the junction can be changed. For example, when the side arm is vertically upward positioned, then in that case gravity will try to keep the liquid phase in the horizontal main pipe, while the gas exits through the side arm. On the other hand, when the side arm is positioned vertically downward, then the gravitational force tries to pull the liquid phase into the side arm, while gaseous phase flows on top in the main pipe. These phenomena can be very well supported by the experiments conducted by Marti and Shoham [4]. They explored the phase separation of stratified wavy flow in a reduced diameter ratio $\mathrm{T}$ junction when its side arm was oriented at different angles with respect to the horizontal plane such as $20^{\circ}, 10^{\circ}, 5^{\circ}, 1^{\circ}, 0^{\circ},-5^{\circ},-10^{\circ},-25^{\circ},-40^{\circ}$, and $-60^{\circ}$. They reported that when the side arm is directed downwards, more liquid flows into the side arm. Whereas, when the side arm was directed upwards, a higher fraction of gas found its way into the branch arm.

According to Griston and Choi [5], inlet liquid volume fraction also affects phase maldistribution at the junction. They reported that less liquid was observed to split to the side branch resulting in higher quality as the inlet liquid volume fraction increased in a uniform diameter T-junction. This is because increasing the inlet liquid volume fraction increases the amount of liquid inside the main pipe at any given moment. This in turn causes the liquid phase inertia to increase, thus higher inertia does not allow liquid phase to turn into side arm. Furthermore, Liu et al. [6] also reported similar findings about phase inertia. They also concluded that in bubble flow condition, gas phase tends to flow into the side arm because the pressure difference of the side arm to main arm is much larger than that for the run arm. Therefore, the lower density gas will respond more easily to pressure gradient than liquid phase for the same pressure force. In addition, Margaris [7] also argued that the separation efficiency in a uniform T-junction depends mainly on phase momentum, the existing flow pattern before the junction, and also on the gravity force distribution in a T-junction. Azzopardi and Rea [8] stated that the degree of phase separation seems to depend on the transit time between liquid-gas mixture and the junction. This means that a slower flow has more time to separate as compared to a fast moving flow. Thus, better phase separation was achieved when the multiphase flow was 
flowing slowly inside the $\mathrm{T}$-junction. It can also be said that in an equal diameter ratio $\mathrm{T}$ junction, multiphase flow has more axial distance and time to jump into side arm, compared to when the diameter ratio of the T-junction is reduced. Likewise, Baker [9] also discussed the effect of a reduced diameter side arm. He stated that a reduction in the side arm diameter has two different effects which are the associated pressure drop, and the axial distance available for take-off. According to Wren [10], the main difference between a reduced and a regular T-junction is the pressure redistribution around the junction. For the same inlet conditions, the reduced side branch causes a higher pressure drop as compared to the uniform T-junction. This is mainly due to the higher gas phase velocities encountered in the reduced side arm for the same fraction of inlet gas flowing down the side arm.

Wren et al. [11] addresses that much of the liquid film that is dragged by the gas towards the side arm may only arrive at the side wall after passing the opening of the reduced side arm. Thus, there is a reduced fraction of liquid take off at side arm. Griston and Choi [5] also reported that reduced side arm diameter decreased the percentage of liquid split to the side branch. This is because, liquid has lesser tendencies to flow through the restricted cross-sectional area of the side arm. They also stated that the percentage of liquid split to the side arm decreased further as the inlet vapor velocity was decreased. Furthermore, Azzopardi [12] also concluded that the effect of diameter ratio is strongest at lower gas rates and least at higher gas and lower liquid flow rate conditions. As stated earlier, previous literature suggests that there exists no generalized phase separation prediction model to this date. This highlights the need for more exploration of different parameters that control the phase separation capabilities of a T-junction. Hence, this paper addresses the effects of diameter ratio, inlet gas saturation, mass split ratio, velocity ratio, side arm angle from the horizontal plane, and length ratio on the amount of gas escaping through the side arm of a T-junction. Numerical simulations are performed on a wide range of operating conditions to generalize the gas extraction trends resulting from the above stated parameters.

\section{METHODS AND MATERIALS}

Eulerian multiphase model was used to conduct this present study. The reason for using this model is because it specializes in modelling immiscible interacting phases. The governing equations for this model as presented by Liu et al. [6] can be summarized as follows:

Two-phase continuity equation:

$$
\frac{\partial}{\partial t}\left(\alpha_{i} \rho_{i}\right)+\nabla \cdot\left(\alpha_{i} \rho_{i} \mathbf{v}_{i}\right)=0
$$

Two-phase momentum balance:

$$
\frac{\partial\left(\alpha_{i} \rho_{i} \mathbf{v}_{i}\right)}{\partial t}+\nabla \cdot\left(\alpha_{i} \rho_{i} \mathbf{v}_{i} \cdot \mathbf{v}_{i}\right)=S
$$

where, $S$ is the source term, which can be written as:

$$
S=-\alpha_{i} \nabla p+\nabla \boldsymbol{\tau}+\alpha_{i} \rho_{i} \mathbf{g} \pm \mathbf{R}_{i, j}
$$


Here, $\mathbf{v}$ is the phase velocity, $\alpha$ is the volume fraction, $\rho$ is the density, $p$ is the hydrostatic pressure, $\mathbf{g}$ is the gravity vector, $\mathbf{R}$ is the interfacial interaction force between two phases, and $\tau$ is the stress tensor of the constituents. The subscript $i$ refers to either liquid $(l)$ or gas $(g)$.

The development of a numerical model of a T-junction for two-phase separation is based on the schematic T-junction in Figure 1, where it consists of the main arm (1), run arm (2), and side arm (3), with symbols indicating the applied inlet/outlet conditions and geometric parameters considered in the present study. The software used for this simulation was ANSYS Fluent. Other parameters such as the velocity inlet for water and gas, inlet gas saturation, flow rate weighting factor for both outlets, and others were taken into consideration. For defining the flow properties at the inlet of the T-junction, Velocity Inlet boundary condition was used. This boundary condition is capable of defining separate superficial velocities for both the phases. Likewise, it is also able to define the volume fraction of the secondary phase. For both outlets of the T-junction, outflow boundary condition was used because it enables one to define the mass split ratio between the two outlets. SIMPLE scheme was used to define pressure-velocity coupling for this model.
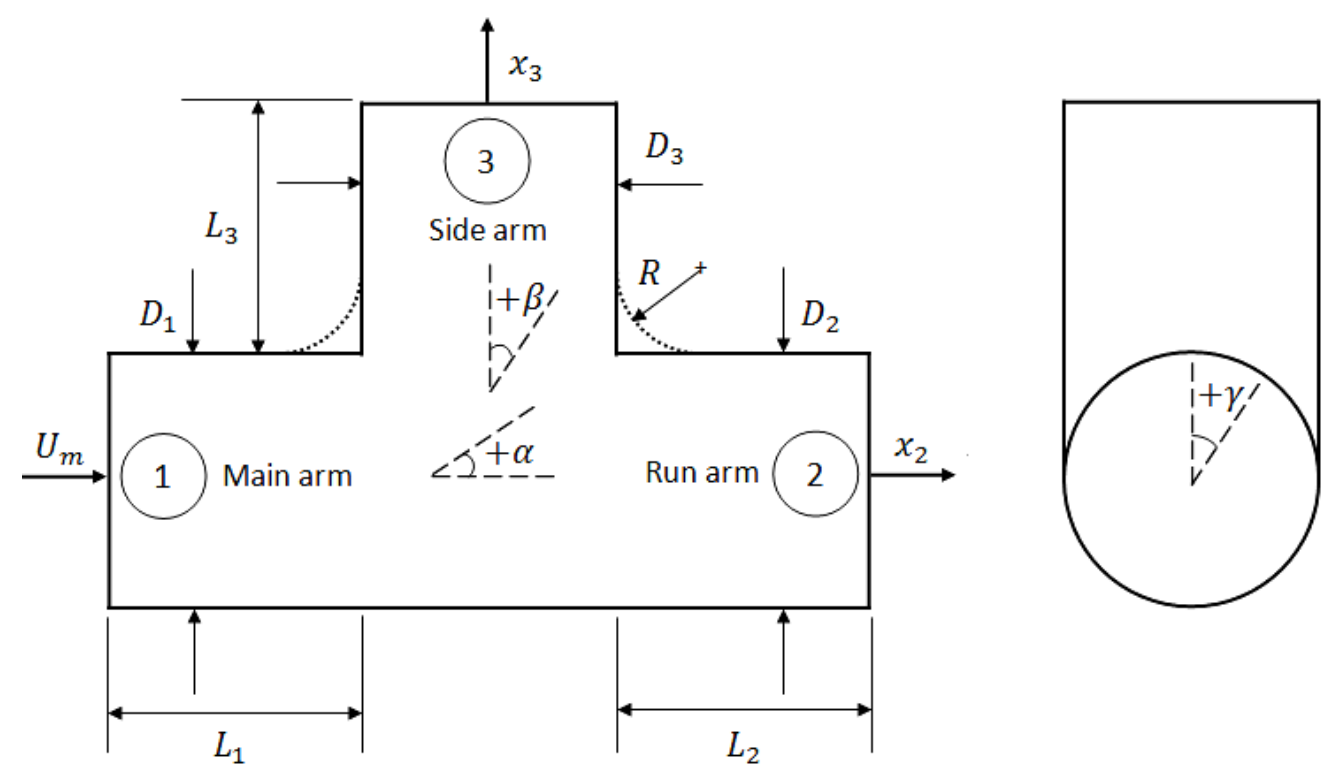

Figure 1. Schematic T-junction with applied inlet/outlet conditions.
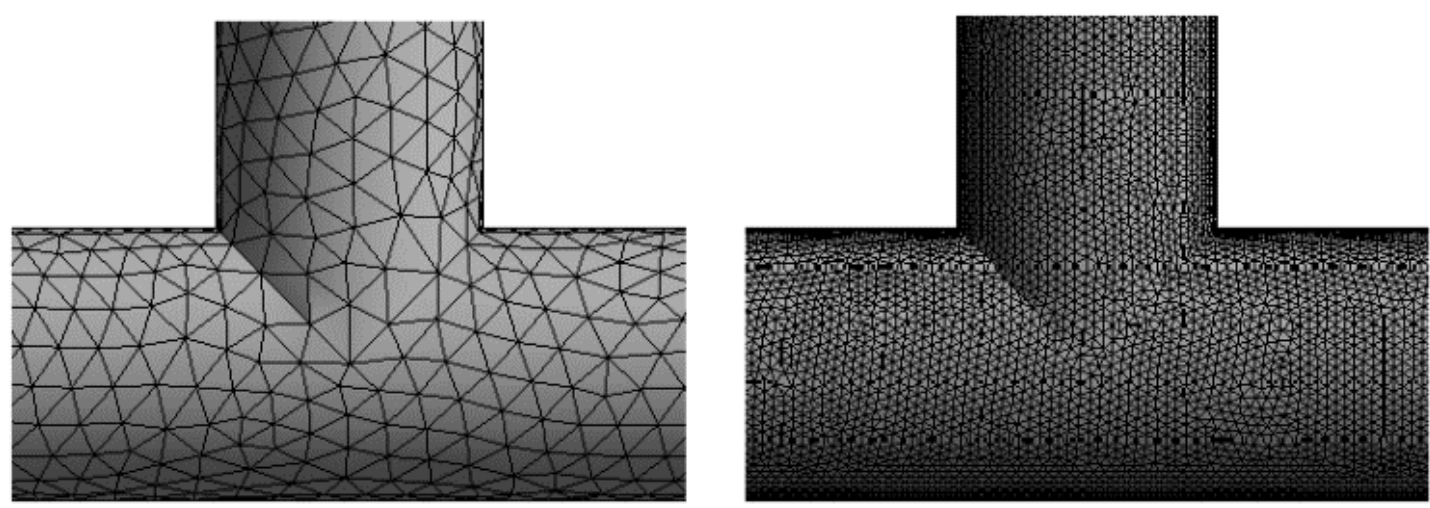

Figure 2. Course model with 9630 voxel (left) and fine model with 37227 voxel (right). 
In order to ensure that the numerical model is mesh independent, mesh dependency and convergent analysis were investigated. ANSYS Fluent default meshing modular was used to create the different meshes for this study. For this analysis, pressure distribution of gas inside the T-junction was chosen to observe the behaviour of the present model upon changing the mesh count. Simulations were performed for six different mesh element sizes. Figure 2 illustrates the lowest and highest contrast voxel model used for the present study. Figure 3 shows the convergence of air pressure when plotted against the mesh density per unit volume. Consequently, a mesh density of 4.77 voxels per unit volume and beyond is used as the criteria to generate the mesh in the model.

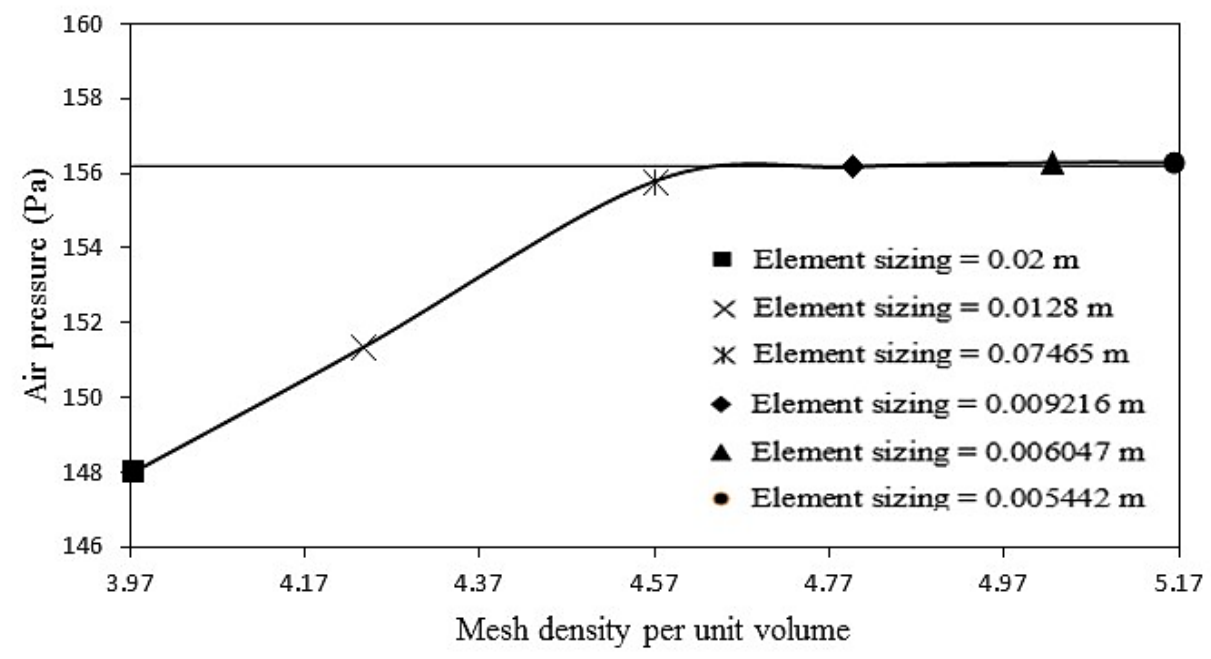

Figure 3. Pressure convergence versus mesh density per unit volume.

Figure 4 shows the contour of air pressure distribution between the coarse mesh and the fine mesh. It is quite evident from this figure that by increasing the fineness of the mesh, the air pressure distribution inside the T-junction becomes much clearer and smooth. In order to understand this concept, consider the pressure distribution at the junction where side arm meets the main pipe. In the contour of coarser mesh, it can be seen that the pressure is experiencing a 'zig-zag' pattern at this location. However, in the contour of finer mesh, the pressure distribution is much smoother.
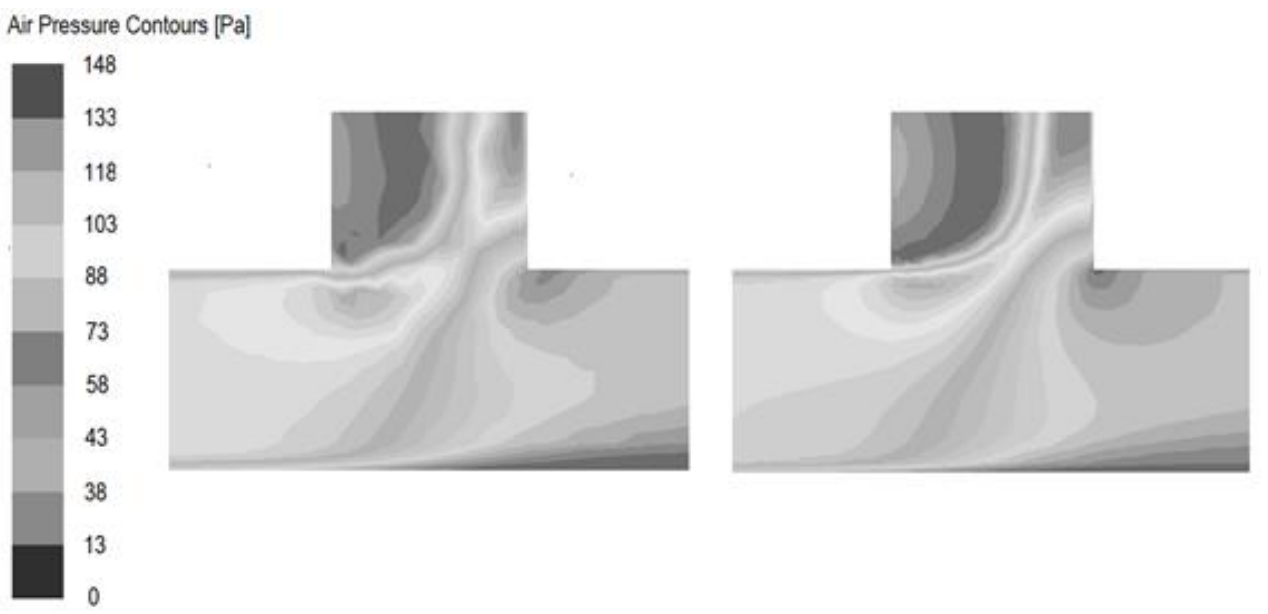

Figure 4. Air pressure contours with coarse (left, 9630 voxel) and fine model (right, 37227 voxel). 


\section{RESULTS AND DISCUSSION}

\section{Validation of Model with Experiments}

The first benchmark used for the validation of present model was from Hong's [13] experimental study. He investigated the phase separation of air and water annular and wavy flow into the side arm of a $9.525 \mathrm{~mm}$ equal diameter ratio $\mathrm{T}$-junction. By conducting extensive experimentation, he tried to study the effect of gas and liquid flow rate, side arm orientation, and inlet flow regime on two-phase separation at the junction. He varied the position of the side arm from $+90^{\circ}$ to $-90^{\circ}$ and thus concluded that side arm orientation plays a very important role in phase separation at the junction. Likewise, he found that inlet flow regime does not have any obvious impact on phase separation. The experimental data also showed that any increase in liquid flow rate would cause the fluid stream in side arm to get richer in gas. This happens because at higher flow rates, liquid has more inertia, hence it flows straight into run arm. On the other hand, when the gas flow rate is increased, the flow stream exiting through the side arm becomes richer in liquid. This is because when more gas turns into side arm, it creates more centrifugal force at the junction. This centrifugal force in return creates higher pressure drop at the junction and inside the branch arm. Therefore, more liquid is sucked into the side arm. The experiments conducted by Hong [13] were performed with extremely low initial gas saturation of $0.085 \%$ and this represents a great challenge using numerical model to reproduce his results.
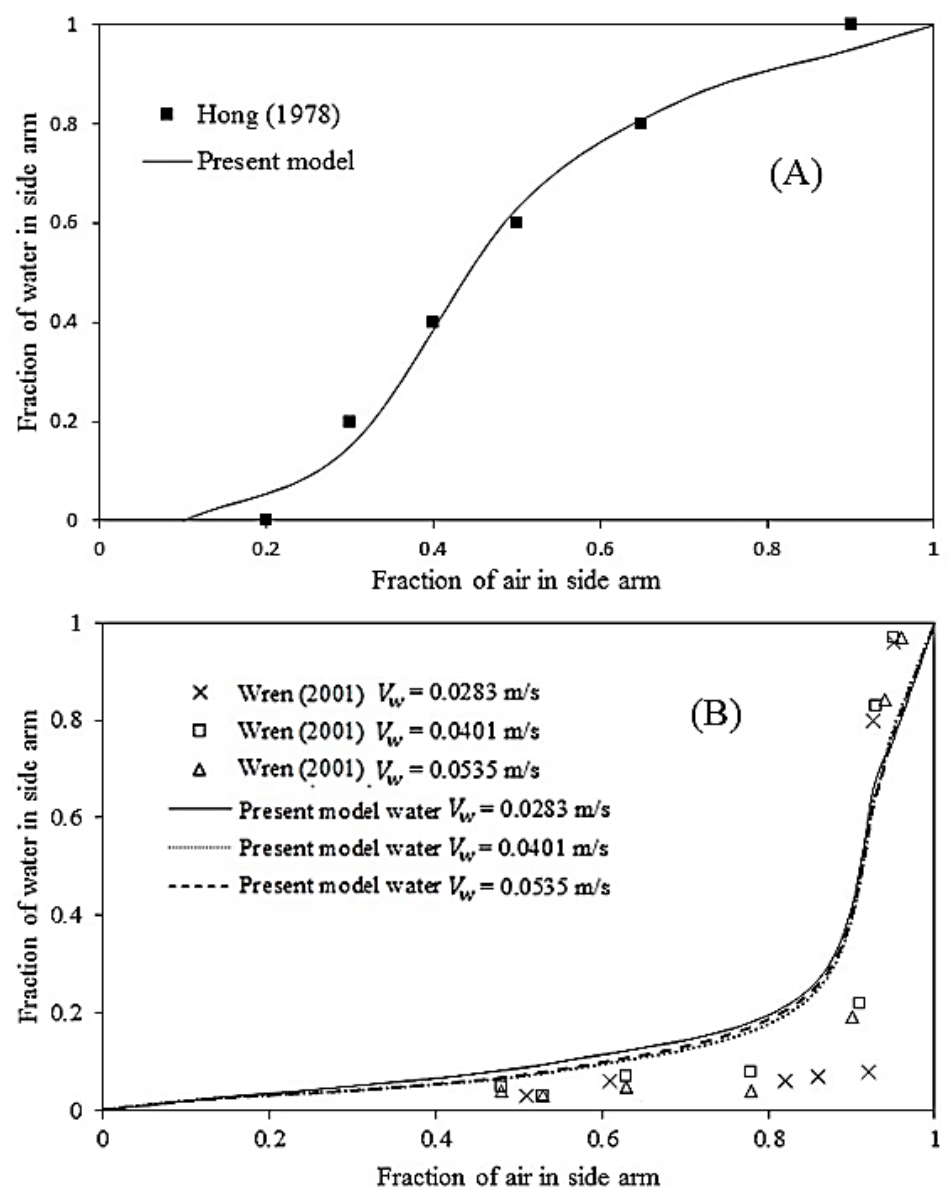

Figure 5. Comparison of present numerical results with (A) Hong [13], and (B) Wren [10] experimental results for a vertically upwards side arm T-junction. 
Figure 5 shows the validation of the present model in comparison to Hong's [9] data for a 12 o'clock side arm configuration. The present model faced difficulties to reproduce the experimental results for side arm pointing at 3 and 6 o'clock positions, but efforts are still underway to investigate this further. The second validation example is taken from the experimental data of Wren [10]. In her experimental research, she analysed the phase separation capabilities of different diameter ratio (1 and 0.6) T-junctions with the side arm oriented at different angles from the horizontal plane. She also focused on the effects of a combination of two T-junction as well as inserts and bends after the Tjunction on phase separation. Her experiments covered a range of gas and liquid superficial velocities falling under the spectrum of stratified and annular flow regimes. From this extensive study, she concluded that inlet flow regime has a great effect on phase separation. Likewise, she reported that in most of the cases, reduced diameter ratio has a positive effect on phase separation. Figure 5 shows the comparison of experimental results and present model. Even though a perfect match is impossible, the close proximity of the present results and Wren's data provides a sufficiently strong evidence that the present T-junction model can be used to analyse the behaviour of gas phase volume fraction in the side arm.

\section{Effect of Geometry on Separation}

Table 1 shows the summary of experimental ata for validation. Table 2 also shows the variation of parameters and values used for the present studies. Due to the large number of simulations and results, efforts were made in the present paper to condense the results, firstly by normalizing several parameters into dimensionless ratios, such as side to main arm diameter ratio etc., and secondly, combining the effects of several parameters into a single graph to understand the interaction effects.

Table 1. Summary of experimental data for validation.

\begin{tabular}{lcc}
\hline \multirow{2}{*}{ Input parameters } & \multicolumn{2}{c}{ Validation } \\
\cline { 2 - 3 } & Hong [13] & Wren [10] \\
\hline Diameter, $\mathrm{D}_{1}, \mathrm{D}_{2} \& \mathrm{D}_{3}(\mathrm{~mm})$ & 9.525 & 127 \\
Length, $\mathrm{L}_{1}, \mathrm{~L}_{2} \& \mathrm{~L}_{3}(\mathrm{~mm})$ & 200 & 2000 \\
Liquid density, $\rho_{1}\left(\mathrm{~kg} / \mathrm{m}^{2}\right)$ & 998.2 (water) & $998.2($ water $)$ \\
Gas density, $\rho_{\mathrm{g}}\left(\mathrm{kg} / \mathrm{m}^{2}\right)$ & $1.225($ air $)$ & 1.225 (air) \\
Liquid velocity, $\mathrm{v}_{1}(\mathrm{~m} / \mathrm{s})$ & 0.02339 & $0.0283,0.0401,0.0535$ \\
Gas velocity, $\mathrm{v}_{\mathrm{g}}(\mathrm{m} / \mathrm{s})$ & 27.432 & 12 \\
Elevation angle of side arm, $\gamma\left(^{\circ}\right)$ & 90 & 90 \\
Initial gas saturation & 0.00085 & 0.002353 \\
Side arm mass split ratio, $\left(\mathrm{x}_{3}\right)$ & $0.1,0.3,0.5,0.7,0.9$ & $0.1,0.3,0.5,0.7,0.9$ \\
\hline
\end{tabular}

Figure 6 shows the response of gas fraction in side arm at different length ratio, diameter ratio, and angle for fixed velocity ratio, gas saturation, mass split ratio at side arm, and bubble ratio. The highest gas fraction in side arm is achieved when $\gamma=90^{\circ}$ or when the side arm is pointing at 12 o'clock position. The lowest gas fraction occurs when $\gamma=-90^{\circ}$ or when the side arm points at 6 o'clock. For $\gamma=90^{\circ}$, the gas fraction in side arm tends to decrease by increasing the length ratio and an opposite effect was observed for $\gamma=-90^{\circ}$, i.e. any increase in length ratio caused more gas to enter the side arm. This is because when $\gamma=90^{\circ}$, increase in side arm length will cause the pressure drop in the side arm to distribute, and it will eventually lose its magnitude. Therefore, less gas will get 
extracted in this condition. However, when $\gamma=-90^{\circ}$, increase in the length of the side arm will cause liquid phase to fall under the action of gravity for a longer length. This will increase the velocity of the liquid phase and according to Bernoulli equation, where speed is high, pressure is low. This pressure drop sucks more liquid into the side arm, which eventually creates a vortex at the junction and sucks more gas into the side arm.

Table 2. Summary of parameters investigated and their values.

\begin{tabular}{lc}
\hline Input parameters & Values \\
\hline Diameter of main \& run arm, $D_{1} \& D_{2}(\mathrm{~mm})$ & 200 \\
Diameter of side arm, $D_{3}(\mathrm{~mm})$ & $40,120,200$ \\
Length of main \& run arm, $L_{l} \& L_{2}(\mathrm{~mm})$ & 2000 \\
Length of side arm, $L_{3}(\mathrm{~mm})$ & $400,1200,2000$ \\
Liquid density, $\rho_{l}\left(\mathrm{~kg} / \mathrm{m}^{2}\right)$ & 998.2 (water) \\
Gas density, $\rho_{g}\left(\mathrm{~kg} / \mathrm{m}^{2}\right)$ & 0.6675 (methane gas) \\
Liquid velocity, $v_{l}(\mathrm{~m} / \mathrm{s})$ & 5 \\
Gas velocity, $v_{g}(\mathrm{~m} / \mathrm{s})$ & $1,3,5$ \\
Mass split ratio at side arm, $\left(x_{3}\right)$ & $0.1,0.3,0.5$ \\
Diameter of gas bubble, $D_{b}(\mathrm{~mm})$ & 1 (avg.), $0.6,0.2$ \\
Angle of side arm, $\gamma\left(^{\circ}\right)$ & $-90,0,90$ \\
Gas saturation $\left(\mathrm{G}_{-} \mathrm{s}\right)$ & $0.1,0.3,0.5$ \\
\hline
\end{tabular}

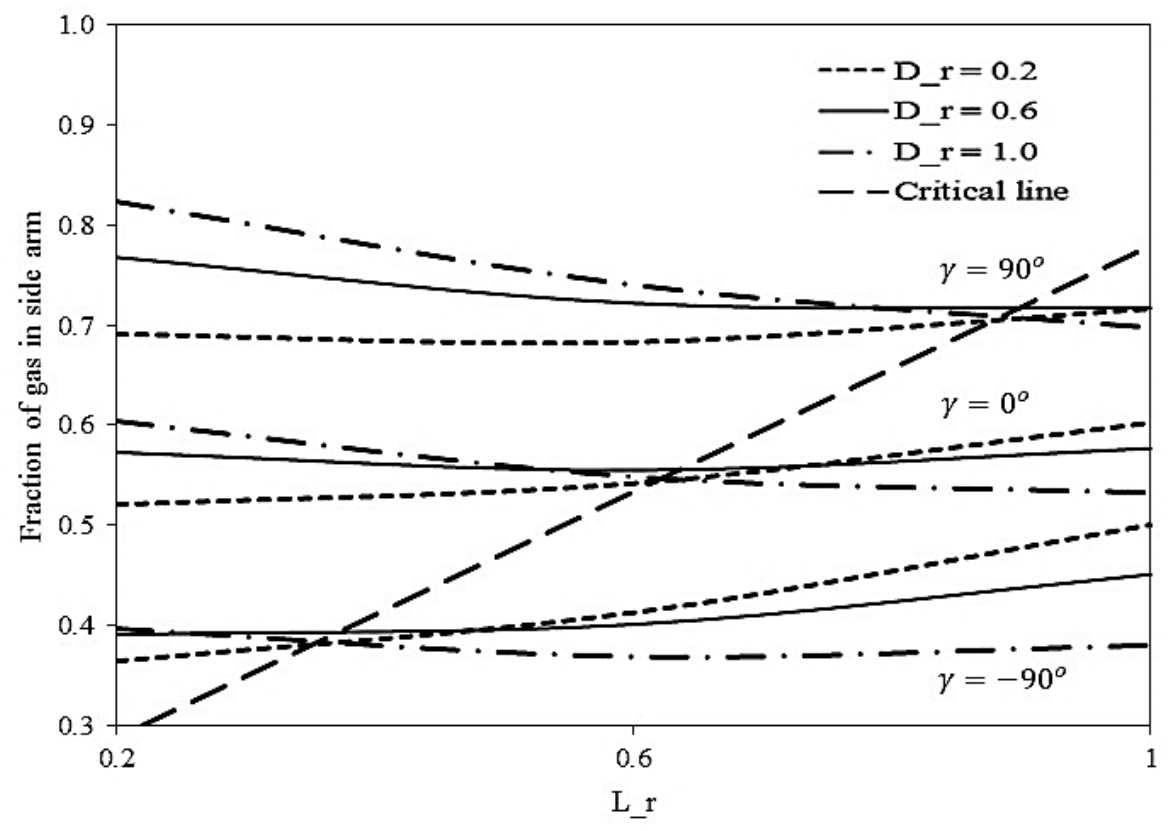

Figure 6. Effect of length ratio, diameter ratio, and angle on phase separation at Tjunction with velocity ratio $=1$, gas saturation $=0.5$, mass split ratio at side arm $=0.5$, and bubble diameter ratio $=1$.

Large diameter ratio is preferable in achieving high gas fraction in side arm when $\gamma=90^{\circ}$. It is quite logical to understand that when the diameter of the side arm is large, then the gas take off will be much easier, due to the gaseous phase's more axial length and time to turn into side arm, as stated earlier. When the side arm is pointing downward, larger diameter ratio will degrade the performance of phase separation in a T-junction. This happens because in this configuration, more liquid inside tries to exit through the 
side arm. Thus, most of the gas will keep flowing into the main arm. For $\gamma=0^{\circ}$, i.e. when the side arm is pointing either at 3 or 9 o'clock position, high gas fraction in side arm can only be achieved in a larger diameter ratio when the length ratio range is between 0.2 to 0.6. Chen et al. [14] also studied the effect of side arm length on two-phase separation. They reported that increasing the length of side arm does enhance the phase separation at the junction but overall, it does not have a very strong influence on the phase separation. A possible reason behind this phenomenon is that when a side arm is positioned at 3/9 o' clock, liquid phase only requires a small hydraulic jump to get into the side arm. Therefore, in cases of smaller diameter ratio, most of the side arm is filled with liquid phase which results in less gas extraction. On the other hand, if the side arm diameter is considerably large, then more gas can pass through it at any given time. Likewise, if the side arm length is too long, then liquid phase starts getting accumulated inside it due to wall resistance and liquid viscosity which will ultimately reduce the amount of gas inside the branch arm. Whereas, if the side arm length is too short, then at certain times the side arm will get choked with liquid phase, as in this case the liquid does not get enough time to maintain its level after entering the side arm due to its very short length.

Furthermore, there is one possible intersection point to be observed between the lines of different diameter ratios for each angle. On this intersection point, the gas fraction in side arm for three different diameter ratios are almost identical and increases from $\gamma=-90^{\circ}$ to $90^{\circ}$. The gravitation force always tries to keep the liquid at the bottom of the main pipe, when it is along the horizontal plane $[3,4]$. Thus, if the position of the side arm is below horizontal plane, then more liquid is sucked into the branch arm. While, if the side arm is oriented to any position above the horizontal plane, then more gas tries to flow into the side arm and most of the liquid phase stays in the main pipe. By analysing the intersection point of each angle, the critical line is drawn by joining each of the points. This critical line is the locus which simply means that the intersection point of other angles are predicted to move along this critical line.

\section{Effect of Operating Conditions on Separation}

Figure 7 illustrates the response of gas fraction in side arm for different velocity, mass split ratios, gas saturation, and bubble ratio for a fixed angle of $90^{\circ}$, diameter ratio, and length ratio. Among these different mass split ratio at side arm, the highest gas fraction in side arm was achieved when the mass flow rate splits equally between side and run arms. The performance of T-junction starts to reduce as mass split ratio at side arm was reduced. The results reported by Pao et al. [15] and Saieed et al. [16], very well supports this finding. The main reason behind this phenomenon is that if less mass splits into the side arm, then it means that most of the mass of the phases rushed straight into the run arm. Thus, less separation takes place in this condition. The increase of velocity ratio slowly reduces the gas fraction in side arm but these nearly flat lines do not show significant changes in terms of gas fraction in side arm. This implies that the effect of velocity ratio on the efficiency of phase separation in T-junction is not significant [17]. For a certain flow regime, any increase in gas superficial velocity has a negative effect on phase separation. While, any increase in liquid superficial velocity enhances phase separation. However, as the overall flow regime is not changing, therefore the phase separation more or less follows the same phase separation trend for any superficial phase velocity combination. For three different mass split ratios, it is obvious that the lower gas saturation results in higher gas fraction in side arm. The gas fraction in side arm tends to converge to a single point for different gas saturation when the value of velocity ratio is approaching to 1 . 


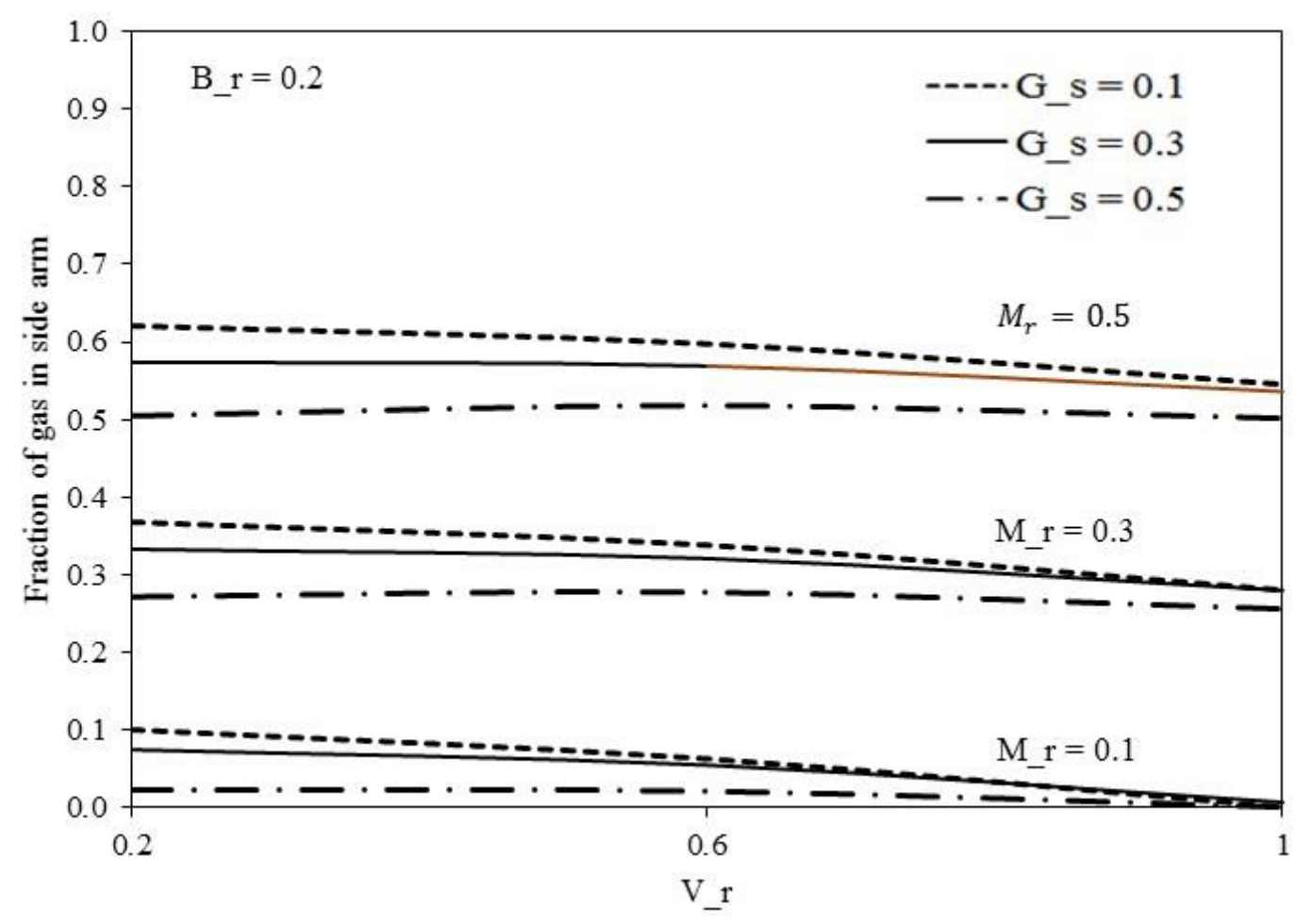

Figure 7. Effect of velocity ratio, gas saturation, mass split ratio and bubble diameter ratio on phase separation at T-junction with side arm pointing at 12 o'clock position.

Fixed diameter and length ratio.

\section{Sensitivity of Parameters}

Figure 8 summarizes the effects on phase separation of all the parameters discussed in this paper, in terms of their relative weighting factors so that they can be quantified in terms of percentage. It can be seen in Figure 8 that the level of influence on phase separation of each parameter is increasing from left towards right starting with angle $(\gamma)$, and followed by velocity ratio, diameter ratio, bubble ratio, length ratio, gas saturation, and mass split ratio at side arm. This arrangement concludes that the mass split ratio at side arm has the highest impact on the phase separation at T-junction, while the angle $(\gamma)$ has the least impact. This also implies that velocity ratio, length ratio, and diameter ratio have comparable effects on the phase separation in a T-junction. Figure 8 suggests that much attention is needed on the bubble ratio, gas saturation, and mass split ratio at side arm because these three parameters dominate the phase separation in a T-junction.

Figure 9 shows the maximum achievable gas volume fraction in side arm versus different optimum ratios of parameters with fixed side arm pointing at 12 o'clock and equal mass split ratio. There are several interesting trends that require attention here. The gas taken-off in the side arm is proportional to the side to main arm diameter ratio, but inversely proportional to the velocity, length ratio, and initial gas saturation. Even though the smallest diameter ratio tested in the present study was 0.2 (see Table 2), however, the results in Figure 9 shows that the maximum allowable gas taken-off starts at a minimum diameter ratio of 0.6. Probably the most surprising result is that the gas to liquid velocity ratio is inversely proportional to the gas volume fraction in the side arm. One possible explanation is that higher gas velocity creates more pressure drop in the side arm which causes more liquid to jump into the side arm. 


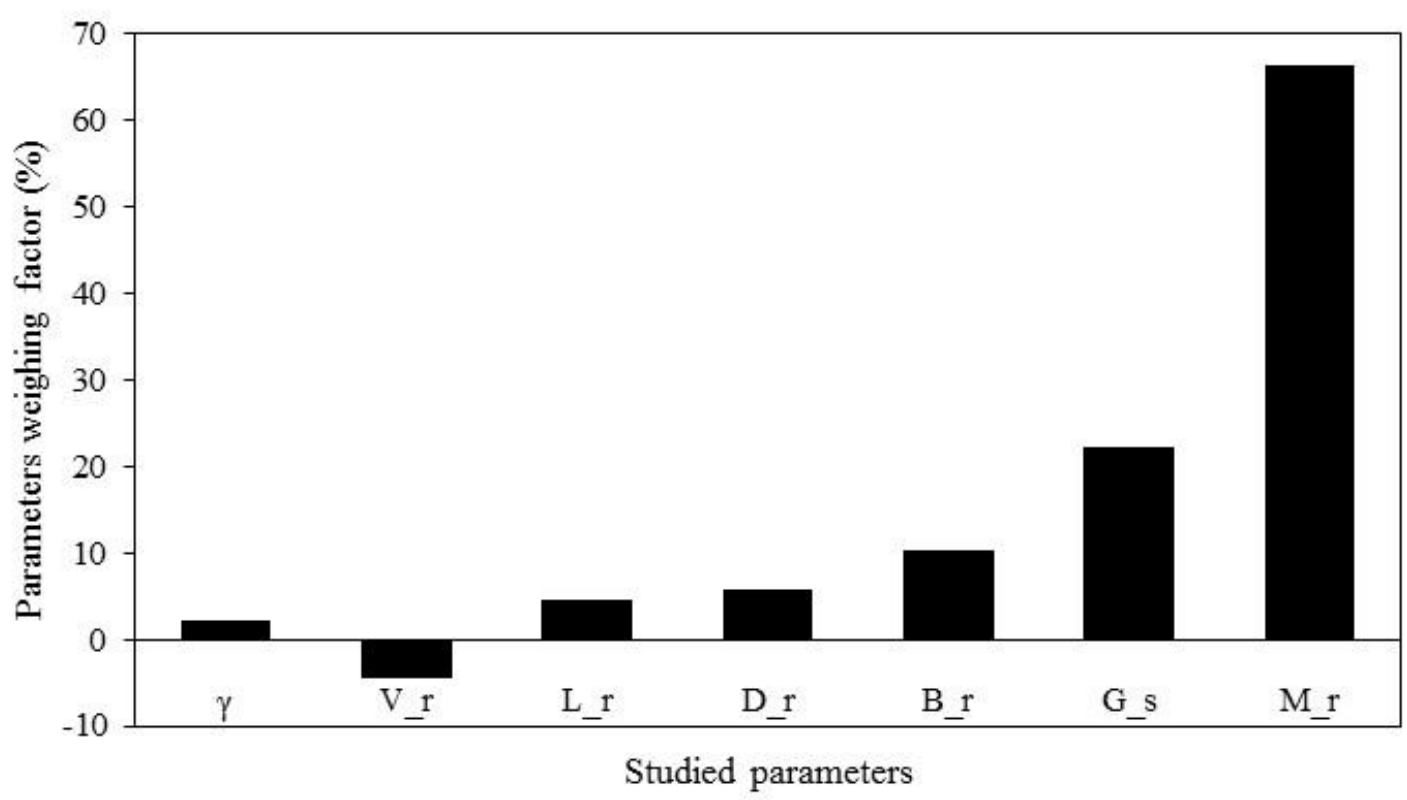

Figure 8. Parameters' weighting factor on two-phase separation in T-junction.

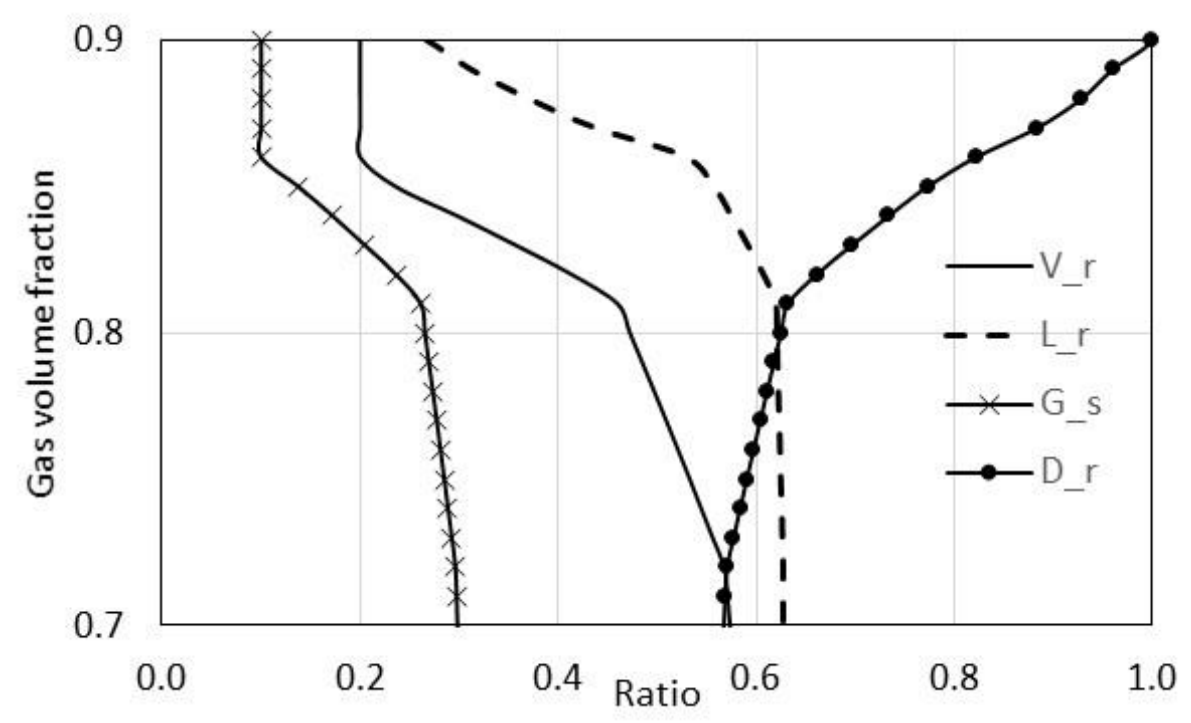

Figure 9: Gas volume fraction in side arm versus different parameters' ratio with fixed angle $=90^{\circ}$ and equal mass split ratio.

\section{CONCLUSIONS}

A numerical study is carried out on a commercial computational fluid dynamics software, in order to understand the effects of different geometrical and flow parameters on twophase separation in a T-junction. The results were converted into different ratios such as diameter ratio, length ratio, velocity ratio, and mass split ratio, in order to present them in a more quantitative form for better understanding. Based on the weighting factor and sensitivity chart, all of these factors were sorted in terms of their degree of affecting the efficiency of phase separation. It was found that mass split ratio has the strongest impact 
on phase separation, while the side arm orientation $(\gamma)$ has the least. Through this information, the behaviour of the two-phase flow in a T-junction can be estimated, so that the phase separation in a T-junction can be controlled based on the individual application.

Besides the abovementioned parameters, there are many other parameters such as angle between run and side arm $(\beta)$, surface roughness, pressure at the inlet and both outlets, radius of curvature along the side arm, and phase viscosities which control the phase splitting behaviour at the junction. Hence, it is recommended to conduct future research on these parameters. Likewise, the effect of flow pattern on multiphase separation still remains unclear as each flow pattern has different impacts on phase splitting behaviour. With the sufficient information, a better design of the T-junction can be implemented so that it can serve as a gas-liquid separator in the oil and gas industry.

\section{ACKNOWLEDGEMENTS}

The authors acknowledge Universiti Teknologi PETRONAS for providing a platform for conducting this research. Special thanks to MOHE for financially supporting this project via FRGS 0153AA-L03 and PETRONAS Research Foundation via YUTP grant 0153AA-E03.

\section{REFERENCES}

[1] Rea S, Azzopardi B. The split of horizontal stratified flow at a large diameter Tjunction. Chemical Engineering Research and Design. 2001;79:470-6.

[2] Lahey R. Current understanding of phase separation mechanisms in branching conduits. Nuclear Engineering and Design. 1986;95:145-61.

[3] Bergman DF, Tek MR, Katz DLV. Retrograde condensation in natural gas pipelines: American Gas Association; 1975.

[4] Marti S, Shoham O. A unified model for stratified-wavy two-phase flow splitting at a reduced T-junction with an inclined branch arm. International Journal of Multiphase Flow. 1997;23:725-48.

[5] Griston S, Choi JH. Two-phase flow splitting at side-branching tees. SPE Western Regional Meeting: Society of Petroleum Engineers; 1998.

[6] Liu Y, Li W. Numerical simulation on two-phase bubbly flow split in a branching T-junction. International Journal of Air-Conditioning and Refrigeration. 2011;19:253-62.

[7] Margaris DP. T-junction separation modelling in gas-liquid two-phase flow. Chemical Engineering and Processing: Process Intensification. 2007;46:150-8.

[8] Azzopardi B, Rea S. Phase separation using a simple T-junction. SPE Annual Technical Conference and Exhibition: Society of Petroleum Engineers; 2000.

[9] Baker G. Separation and control of gas-liquid flows at horizontal T-junctions: University of Nottingham; 2003.

[10] Wren EMK. Geometric effects on phase split at a large diameter T-junction: University of Nottingham; 2001.

[11] Wren E, Baker G, Azzopardi B, Jones R. Slug flow in small diameter pipes and T-junctions. Experimental Thermal and Fluid Science. 2005;29:893-9.

[12] Azzopardi B. The effect of side arm diameter on phase split at T-junctions. SPE annual technical conference 1999.

[13] Hong K. Two-phase flow splitting at a pipe tee. Journal of Petroleum Technology. 1978;30:290-6. 
[14] Chen JL, He LM, Luo XM, Bai HT, Wei YH. Simulation of oil-water two phase flow and separation behaviors in combined $\mathrm{T}$ junctions. Journal of Hydrodynamics, Ser B. 2012;24:848-57.

[15] Pao W, Hashim FM, Ming LH, Ramli MF, Junoh AK, Roslan N, et al. Numerical investigation of gas separation in T-junction. AIP Conference Proceedings: AIP Publishing; 2015. p. 070001.

[16] Saieed A BS, Pao W, Hashim FM, Norpiah RBM. A review of T-junction geometrical effect on two-phase separation. ARPN Journal of Engineering and Applied Sciences 2016;11:14233-8.

[17] Conte G, Azzopardi B. Film thickness variation about a T-junction. International Journal of Multiphase Flow. 2003;29:305-28. 